Proyecciones

Vol. 19, No 2, pp. 197-205, August 2000.

Universidad Católica del Norte

Antofagasta - Chile

\title{
THE PERIOD FUNCTION IN A CLASS OF QUADRATIC KOLMOGOROFF SYSTEMS *
}

\author{
MARCO URIBE S. \\ Univ. Católica de la Santísima Concepción, Chile \\ and \\ MYRNA WALLACE \\ Universidad de Concepción, Chile
}

\begin{abstract}
In this paper we consider the family of quadratic Kolmogoroff systems with a center in the real quadrant:

$$
\left\{\begin{array}{l}
\dot{x}=x(1-x-a y) \\
\dot{y}=y(-1+a x+y)
\end{array},\right.
$$

where $1<a<\infty$. This system has three invariant lines (the coordinate axes and the line $x+y-1=0$ ) and a family of periodic solutions nested around a center and filling out the triangle determined by the three invariant lines. Using integrability of this system we reduce the abelian integral representing the period function and its derivative. The main result is that the corresponding period function is monotone increasing for values of the parameter near $a=3$.
\end{abstract}

*Partially supported by a Grant of Universidad de Concepción P.I. 98.015.0111.0 


\section{Introduction}

A Kolmogoroff system is given by a planar differential system of the form

$$
\left\{\begin{array}{l}
\dot{x}=x F(x, y) \\
\dot{y}=y G(x, y)
\end{array}\right.
$$

with $F, G$ of $C^{1}$ class.

This kind of system appears usually in predator-prey models and so only dynamics on the first quadrant is considered. In this paper we consider the quadratic Kolmogoroff system:

$$
\left\{\begin{array}{l}
\dot{x}=x(1-x-a y) \\
\dot{y}=y(-1+a x+y)
\end{array},\right.
$$

where $1<a<\infty$.

A system of this class has a family of periodic solutions nested around a center at $\left(\frac{1}{1+a}, \frac{1}{1+a}\right)$ and filling out the triangle formed by the saddle points $(0,0),(1,0)$ and $(0,1)$. The family of periodic solutions forms a center period annulus

The function which associates to any closed curve its period, is called the period function. We are interested in obtaining the global description of the period function defined on the center period annulus.

In section 2 , we analyze the period function and we prove that it is monotone increasing for $a=3$.

In section 3 we prove, by means of a linear change of coordinates that system (1.1), in the Bautin's form is of type $B_{2}$ for all $a>1$ Bautin's systems and their period functions were defined in [1] by $\mathrm{C}$. Chicone and M. Jacob where they conjectured that for $B_{2}$ systems the period function is globally monotone increasing.

By calculating the first three periodic coefficients for $a>1$, we realize that they are alternatively positive and negative. This result suggests us that there would exists a value of the parameter $a$ such that the corresponding system has a period function with critical points.

In general, aside from their intrinsic interest, monotonicity properties of the period function are important in the question of existence 
and uniqueness of autonomous boundary value problems [2], in the study of subharmonic bifurcation of periodic oscillations, and in the analysis of the problem of linearization.

\section{The Period Function}

System (1.1) has a first integral

$$
H(x, y)=x y(1-x-y)^{a-1}
$$

In fact

$$
\begin{aligned}
\frac{d H}{d t}= & H_{x} \dot{x}+H_{y} \dot{y}=H_{x} x(1-x-a y)+H_{y} y(-1+a x+y) \\
= & x y(1-x-y)^{a-2}[(1-y-a x)(1-x-a y)+ \\
& (1-x-a y)(-1+a x+y)] \\
= & 0
\end{aligned}
$$

for all $t \in \mathbf{R}$.

Since $H(x, y)=0$ for $(x, y)$ on the invariant triangle $x=0, y=0$, $x+y=1$ and $H\left(\frac{1}{1+a}, \frac{1}{1+a}\right)=(a-1)^{(a-1)}(a+1)^{-(a+1)}$, the closed orbit $\gamma(E)$ surrounding the center $\left(\frac{1}{1+a}, \frac{1}{1+a}\right)$ is defined by $H(x, y)=E$, where $0<E<(a-1)^{(a-1)}(a+1)^{-(a+1)}$.

The period of this orbit is given then by the formula

$$
T(E)=\int_{\gamma(E)} \frac{d x}{x(1-x-a y)} .
$$

We are interested in the study of $T$ as a function of the energy $E$.

We observe that for $E$ going from 0 to $(a-1)^{a-1}(a+1)^{-(a+1)}$, the closed orbit goes from the polycycle to the center so that a negative derivative $\frac{d T}{d E}$, indicates an increasing period function.

In order to calculate $\frac{d T}{d E}$, we introduce the change of variables

$$
u=x y \quad \text { and } \quad v=1-x-y .
$$


on a periodic orbit $\gamma(E): u=E v^{1-a}$ and $\gamma(E)$ can be given by $\left(x_{+}(v), y_{-}(v)\right)$ and $\left(x_{-}(v), y_{+}(v)\right)$ where

$$
\begin{array}{ll}
x_{+}(v)=y_{+}(v)=\frac{1}{2}(1-v)+\frac{1}{2} \sqrt{(1-v)^{2}-4 E v^{1-a}}, & v_{\min }<v<v_{\max } \\
x_{-}(v)=y_{-}(v)=\frac{1}{2}(1-v)-\frac{1}{2} \sqrt{(1-v)^{2}-4 E v^{1-a}}, & v_{\min }<v<v_{\max }
\end{array}
$$

and $v_{\min }$ and $v_{\max }$ are zeroes of $v^{a-1}(1-v)^{2}=4 E$, with $0<v_{\min }<$ $v_{\max }<1$. Using $(2.2)$ the integral $T(E)$ given by (2.1) can be written in the new coordinates $u$ and $v$. Since $u$ and $v$ are related to each other on $\gamma(E), T(E)$ can be rewritten as an integral in $v$ alone. Using the simmetry of $\gamma(E)$, the integral $T(E)$ becomes

$$
T(E)=2 \int_{v_{\min }}^{v_{\max }} \frac{d v}{v \sqrt{(1-v)^{2}-4 E v^{1-a}}}
$$

Now, we consider new a change of variables. Define

$$
h(v)=\left\{\begin{array}{cl}
\left(v-v_{0}\right) \sqrt{\frac{w_{o}-g(v)}{\left(v-v_{0}\right)^{2}}} & v \neq v_{0} \\
0 & v=v_{0}
\end{array}\right.
$$

where

$$
g(v)=v^{a-1}(1-v)^{2}, v_{0}=\frac{a-1}{a+1}, w_{0}=g\left(v_{0}\right)=4 \frac{(a-1)^{a-1}}{(a+1)^{a+1}}
$$

Since $g$ has a quadratic maximum at $v_{0}, h$ is a smooth function. Moreover, it is easy to see that $h^{\prime}(v)>0$, for all $v_{\min }<v<v_{\max }$, so $h$ monotone increasing. Setting $r=h(v)$, the integral $T(E)$ can be simplified to

$$
T(E)=2 \int_{-\sqrt{w_{0}-4 E}}^{\sqrt{w_{0}-4 E}} \frac{d r}{h^{\prime}\left(h^{-1}(r)\right)\left(h^{-1}(r)\right)^{\frac{3-a}{2}} \sqrt{\left(w_{0}-4 E\right)-r^{2}}}
$$


This form of the integral suggests the substitution $r=\sqrt{w_{0}-4 E} \sin \theta$.

We obtain

$$
T(E)=2 \int_{-\frac{\pi}{2}}^{\frac{\pi}{2}} \frac{d \theta}{h^{\prime}\left(h^{-1}\left(\sqrt{w_{0}-4 E} \sin \theta\right)\right)\left(h^{-1}\left(\sqrt{w_{0}-4 E} \sin \theta\right)\right)^{\frac{3-a}{2}}}
$$

In this form the integral can be derivated to obtain

$$
\begin{gathered}
\frac{d T}{d E}=\frac{4}{\sqrt{w_{0}-4 E}} \int_{-\frac{\pi}{2}}^{\frac{\pi}{2}} \frac{h^{\prime \prime}\left(h^{-1}\left(\sqrt{w_{0}-4 E} \sin \theta\right)\right)\left(h^{-1}\left(\sqrt{w_{0}-4 E} \sin \theta\right)+\frac{3-a}{2} h^{\prime}\left(h^{-1}\left(\sqrt{w_{0}-4 E} \sin \theta\right)\right)^{3}\right.}{h^{\prime}\left(h^{-1}\left(\sqrt{w_{0}-4 E} \sin \theta\right)\right)^{3}\left(h^{-1}\left(\sqrt{w_{0}-4 E} \sin \theta\right)\right)^{\frac{5-a}{2}}} \\
(2.8) \\
\sin \theta d \theta
\end{gathered}
$$

Now, a straightforward calculation proves the

Theorem : The period function is monotone increasing in a neighborhood of $a=3$

Proof : If $a=3$ in (2.8), we obtain

$$
\frac{d T}{d E}=\frac{4}{\sqrt{\frac{1}{16}-4 E}} \int_{-\frac{\pi}{2}}^{\frac{\pi}{2}} \frac{h^{\prime \prime}\left(h^{-1}\left(\sqrt{\frac{1}{16}-4 E} \sin \theta\right)\right)}{\left(h^{\prime}\left(h^{-1}\left(\sqrt{\frac{1}{16}-4 E} \sin \theta\right)\right)\right)^{3}} \sin \theta d \theta
$$

We have $h^{\prime}(v)>0$ and $h^{\prime \prime}(v) \sin \theta<0$ for all $\theta$ in $\left[-\frac{\pi}{2}, \frac{\pi}{2}\right]$ ( here we have put $v=h^{-1}\left(\sqrt{\frac{1}{16}-4 E} \sin \theta\right)$ for simplicity of notation ).

In fact

$$
h^{\prime \prime}(v)=\left\{\begin{array}{lll}
-\frac{4\left(v-\frac{1}{2}\right)^{4}\left(2 v^{2}-2 v-1\right)}{\sqrt{\frac{1}{16}-v^{2}(1-v)^{2}}} & \text { for } & v \leq \frac{1}{2} \\
\frac{4\left(v-\frac{1}{2}\right)^{4}\left(2 v^{2}-2 v-1\right)}{\sqrt{\frac{1}{16}-v^{2}(1-v)^{2}}} & \text { for } & v>\frac{1}{2}
\end{array}\right.
$$

and $\left[v_{\min }, v_{\max }\right]=\left[\frac{1}{2}-\frac{\sqrt{1-8 \sqrt{E}}}{2}, \frac{1}{2}+\frac{\sqrt{1-8 \sqrt{E}}}{2}\right] \subset\left[v_{1}, v_{2}\right]$ for $v_{1}, v_{2}=\frac{1}{2} \pm \frac{\sqrt{3}}{2}, \quad$ the roots of $2 v^{2}-2 v-1=0 . \quad$ Then $h^{\prime \prime}(v)>0$ 
for $v_{\min }<v<\frac{1}{2}$ and $h^{\prime \prime}(v)<0$ for $\frac{1}{2}<v<v_{\max }$. It follows that $h^{\prime \prime}(v) \sin \theta \leq 0$ for all $\theta$ in $\left[-\frac{\pi}{2}, \frac{\pi}{2}\right]$, because $h$ is monotone increasing.

This proves that

$$
\frac{d T}{d E}<0 \text { for all } 0<E<\frac{1}{64}
$$

Remark : Since $T(E)$ is a continuous function on the parameter $a$, we can assert that $T(E)$ is monotone increasing for $a$ in a neighborhood of $a=3$.

\section{The Period Function near the center}

In this section we compute the first period constants of system (1.1), using the results of [3], [4] .

First, using the change of variables

$$
X=x-\frac{1}{1+a} \quad ; \quad Y=y-\frac{1}{1+a}
$$

we carry the center to the origin

$$
\left\{\begin{array}{l}
\dot{X}=-\frac{1}{1+a} X-\frac{a}{1+a} Y-a X Y-X^{2} \\
\dot{Y}=\frac{a}{1+a} X+\frac{1}{1+a} Y+a X Y+Y^{2}
\end{array}\right.
$$

Bringing the linear part of (3.1) to the Jordan form we obtain

$$
(3.2)\left\{\begin{array}{llc}
\dot{u} & = & -\frac{\sqrt{a-1}}{\sqrt{a+1}} v-a \sqrt{a^{2}-1} u v \\
\dot{v} & = & \frac{\sqrt{a-1}}{\sqrt{a+1}} u-\sqrt{a^{2}-1} u^{2}-\left(a^{2}+a-2\right) u v+\sqrt{a^{2}-1} v^{2}
\end{array}\right.
$$

where

$$
u=\frac{1}{a} Y \quad ; \quad v=-\frac{1}{\sqrt{a^{2}-1}} X-\frac{1}{a \sqrt{a^{2}-1}} Y
$$


with a rescaling of time $t=\frac{\sqrt{a+1}}{\sqrt{a-1}} T$, the system is transformed into

$$
\left\{\begin{array}{lcc}
\dot{x} & = & -y-a(a+1) x y \\
\dot{y} & = & x-(a+1) x^{2}-(a+2) \sqrt{a^{2}-1} x y+(a+1) y^{2}
\end{array}\right.
$$

where we returned to the initial notations. (3.3) has the general form

$$
\left\{\begin{array}{ccc}
\dot{x} & = & -y+A x y \\
\dot{y} & = & x-B x^{2}+C x y+B y^{2}
\end{array}\right.
$$

which is a special class of Bautin's center $B_{2}$. Systems can be expressed in the complex plane

$$
\dot{z}=z i+z^{2}\left[\frac{C}{4}-\left(\frac{A}{4}+\frac{B}{2}\right) i\right]+\dot{z}^{2}\left[-\frac{C}{4}+\left(\frac{A}{4}-\frac{B}{2}\right) i\right]
$$

and in this form we can compute the first four periodic coefficients

$$
\begin{aligned}
& p_{0}=2 \pi \quad ; \quad p_{2}=\frac{\pi}{2}\left[A^{2}-4 A B+4 B^{2}+C^{2}\right] \\
& p_{4}=\frac{2 \pi}{768}\left[\left(C^{2}+(A+2 B)^{2}\right)\left(C^{2}+(A-2 B)^{2}\right)-\frac{4}{3}\left(C^{2}+(A-2 B)^{2}\right)^{2}\right] \\
& p_{6}=\frac{\pi}{19906560}\left(C^{2}+(A-2 B)^{2}\right)\left(1296\left(C^{2}+(A+2 B)^{2}\right)^{2}-\right. \\
& 4104\left(-C\left(C^{3}-3 C(A+2 B)^{2}\right)+(A-2 B)\left(3 C^{2}(A+2 B)-(A+2 B)^{3}\right)\right)+ \\
& 14976\left(C^{2}+(A+2 B)^{2}\left(C^{2}+(A-2 B)^{2}+33808\left(C^{2}+(A-2 B)^{2}\right)\right)\right.
\end{aligned}
$$

and for (3.3)

$$
\begin{aligned}
& p_{0}=2 \pi \quad ; \quad p_{2}=\frac{\pi}{6} a(1+a)(2+a)^{2} \\
& p_{4}=-\frac{\pi}{288} a^{2}(1+a)^{2}(2+a)^{2}\left(16+16 a+a^{2}\right)
\end{aligned}
$$




$$
\begin{gathered}
p_{6}=\frac{\pi}{311040} a^{2}(1+a)^{3}(2+a)^{2}\left(-8208+63512 a+143440 a^{2}+98040 a^{3}+\right. \\
\left.25294 a^{4}+2003 a^{5}\right)
\end{gathered}
$$

Remark : The sign of the first period constants are

$$
p_{2}>0 \quad ; \quad p_{4}<0 \text { and } p_{6}>0 \text { for all } a>1
$$

\section{References}

[1] Chicone C. and Jacob M. Bifurcation of critical periods for plane vector fields. Trans. Amer. Math. Soc. 312, N 12, (1989).

[2] Chicone C. The monotonicity of period function for planar hamiltonian vector fields. Journal of Differential Equation 69 , (1987)

[3] Gasull A. , Guillamon A. and Mañosa V. An expression for the first Liapunov and period constants with application . Preprint , Universidad Autonoma de Barcelona, España.

[4] Gasull A. , Guillamon A. and Mañosa V. Center and Isochronicity conditions for systems with homogeneus nonlinearities. Proceeding of the $2^{a}$ catalan days on apllied mathematics. (1995)

[5] Van Horssen W and Reyn J. Bifurcation of limit cycles in a particular class of quadratic systems. Differential and Integral Equation Vol. 8, N 4, (1995)

Received : October 1999. 
Marco Uribe S.

Area de Ciencias Básicas

Facultad de Ingeniería

Universidad de la Santísima Concepción

Casilla 297 - V

Concepción

and

\section{Myrna Wallace C.}

Departamento de Matemática

Facultad de Ciencias Físicas y Matemáticas

Universidad de Concepción

Casilla 20 - C

Concepción 